B. Luchsinger: Von d. Venenherzen in d. Flughaut d. Fledermäuse. 445

(Physiologisehes Laboratorium der Thierarzneischule zu Bern.)

\title{
Von den Venenherzen in der Flughaut der Fledermäuse.
}

(Ein Beitrag zur Lellre von dem peripheren Gefässtonus.)

Von

IS. Luchsinger.

Erste Mittheilung.

Im Laufe des verflossenen Sommersemesters bot sich die willkommene Gelegenheit, in einer Vorlesung uber Blutcirculation den von Wharton Jones ${ }^{1}$ ) in der Flughaut der Fledermäuse entdeckten Venenpuls zu demonstriren.

Der Anblick dieser reizenden Erscheinung machte sofort den lebhaften Wunsch rege, die näheren Bedingungen für die Pulsation dieser elementaren Herzen zu ergrïnden. Das Studium solch eigenthümlicher Gefässe bot verlockende Aussicht für die Erkenntniss der viel discutirten aber noch immer nicht geeinigten Lehre von der Innervation der Gefässwand.

Nachdem Cl. Bernard in seiner fundamentalen Entdeckung: endlich in bündigster Weise einen Einfluss des Nervensystems auf die Muskelwand des Gefässrohres dargethan, schienen auf den ersten Blick höchst einfache Vorstellungen zu genügen. Die Durchschneidung des Sympathicus macht Hyperämie, die Reizung Blässe des Ohres. Zwischen Gefässnerv und Ringmuskulatur der Gefässwand aber schienen keine andern Beziehungen zu gelten, als wie zwischen irgend welchem Skeletmuskel und seinem zugehörenden Nerven. Aber schon wenige Jahre hernach sah Sch if f diese Hyper-

1) Wharton Jones, Philosophical Transactions. 1852. 
ämie, diese Erschlaffung der Gefässwand keineswegs andauern, kehrte vielmehr eine neue, oft sogar kolossal entwickelte Blässe des Kaninchenohres zuriick. Diese „Rückkehr des Tonus“ passte nicht zu den engen Vorstellungen, an die man sich so leicht gewöhnt hatte; die Thatsache selber aber hatte beinahe 20 Jahre in dunkler Vergessenheit zu sehmachten.

Eine nicht geringere Schwierigkeit bot eine andere, ebenfalls schon frühzeitig gefundene, aber gern ignorirte Entdeckung. Schiff, Lister, Bernard hatten schon zweifellose Belege für die Existenz aetiv gefảsserweiternder Nerven geliefert, aber es bedurfte jenes energischen Aufschwunges experimenteller Untersuchung, wie er vor wenig Jahren erst auf diesem Gebiete ganz besonders durch Goltz angefacht worden, um endlich die Anerkennung dieser Nerven zu unantastbarem Gemeingut zu machen.

Diese Erweiterung unserer Kenntnisse hatte endlich eine neue Theorie zu veranschaulichen. Eine geistreiche Intuition des alten Senac schien glückliche Führung zu geben. Senac schon hatte die Gefässe als lauter kleine Herzen betrachtet; Lister, Bernard, Goltz u. v. A. verfolgten diesen Gedanken.

Im Herzen selber liegt der Sitz seiner rhythmischen Entladung; in gleichem Sinne scheint auch die Gefässwand eine eigene, meist tonische Erregung in sich selber zu besitzen. Die Rückkehr des Tonus wäre dann nur eine durch den reichlicheren Blutstrom bedingte Kräftigung der ursprünglich schon in der Gefässwand vorhandenen Automatie. Wie im Herzen, so dürften vielleichtauch hier in der Gefässwand gangliöse Elemente die Träger der automatischen Erregung sein. Diese Erregung aber würde in beiden Gebilden durch gewisse Nerven gesteigert, durch andere gehemmt werden können. So wïden die gefässverengenden Nerven in den pulsbeschleunigenden, die gefässerweiternden aber in den hemmenden Nerven des Herzens eine schönste Analogie finden.

Endlich schienen die zuerst von $W$ hart on $J_{0}$ nes an unserem Objecte entdeckten, bald darauf von Schiff, seither von vielen Anderen an verschiedensten Gefässgebieten bestätigten rhythmischen Contractionen des Gefässrobres in schlagendster Weise einer Anschauung das Wort zu reden, die in Herz und Gefässwand auch physiologisch so viele Uebereinstimmung sieht.

So bestechend aber solche Auffassung auch sein mochte, so traten derselben gleichwohl auch wieder mancherlei Bedenken 
entgegen, und versuchten solche im wesentlichen eine Rückkehr zur älteren Lehre.

So erklärt Exner die anfangs so schwierige Deutung der activen Gefässerweiterung aus einer einfachen Leistung der Längsmuskeln des Gefässrohres, kann dabei allerdings nicht umhin, selber die im Vergleich zu dem allgemeinen Vorkommen der Function äusserst spärliche Vertretung von Längsfasern zu bekennen.

Seit weiterhin Schiff an gewissen leicht erregbaren Muskeln des Gesichts und der Zunge einige Tage nach Durchschneidung ihrer Nerven fibrilläre Zuckungen nachgewiesen, war die Rückkehr des Gefässtonus einige Tage nach Durchschneidung der Gefässnerven in ähnlicher Weise zu deuten, durch fibrilläre Zuckungen der entnervten Gefässwand erklärlich genug. Solche Erklärung musste um so eher befriedigen, als die doch auf der andern Seite zumeist postulirten Localganglien allgemeiner histologischer Anerkennung sich noch keineswegs erfreuen.

Endlich hatte schon Schiff die Rhythmik der Gefässcontractionen untersucht, als Quelle dieser rhythmischen Erregung aber ganz im Gegensatz zur neuern Theorie ausschliesslich centrale Ursachen erkannt.

„Wie erwähnt hören diese regelmässigen, alle Ohrarterien gleichzeitig betreffenden rhythmischen Bewegungen nach Durchschneidung der Nerven auf und das grosse mittlere Gefäss scheint erweitert völlig stillzustehen. Betrachtet man es aber bei grossen Kaninchen einige Tage nach der Operation scharf und längere Zeit, so sieht man oft eine einzelne Stelle des erweiterten Gefässes etwas enger werden. Diese vorübergehende Verengerung kann sich an 2-3 Stellen des Gefässes zeigen, während die dazwischen liegenden weit und bluterfüllt sind. Diese Bewegungen sind für die contractile Faserzelle der Arterie ganz dasselbe, was ich frïher bei quergestreiften Muskeln einige Tage nach Durchschneidung ihrer Nerven beobachtet habe" ${ }^{1}$ ).

Bald darauf hatte Schiff auch an unserem Objecte die Sache verfolgt, und gelangte zu gleichem Ergebniss. "Bei Fledermäusen sieht man nach Durchschneidung des Armgeflechts die Gefässe der Flughaut der Arme weiter werden und mehr Blut

1) Sohiff, Arch. f. physiol. Heilk. Bd. XIII. 1854. p. 527. 
aufnehmen. Der von Wharton Jones entdeckte Venenpuls hört auf ${ }^{4}$ ).

In der Folge häuften sich die Untersuchungen, ein neues Object - die Schwimmhaut des Frosches - trat noch hinzu.

Von verschiedenen Seiten, von Gunning, Roever, Huizinga wurde nun geradezu behauptet, dass im Gegentheil schon wenige Tage nach einer Nervendurchschneidung die Rhythmik wiederkehre, wurde also auf locale Ursache derselben geschlossen; von andern Forsehern aber erfolgte ebenso entschiedener Widerspruch.

Die Beobachtungen Roever's bezogen sich auf das Kaninchenohr, es war der Halssympathicus durchschnitten. Da aber in vielen Fällen anch der n. auricularis magnus reichlich vasomotorische Fasern führt, so hätte eben auch dieser durchschnitten sein müssen, was Roever versäumte ${ }^{2}$ ).

Noch in allerletzter Zeit hat dagegen Sigm. Mayer sich wieder vollkommen in Schiff's Sinne ausgesprochen. „Wenn hier und da gelehrt wird, dass die mittlere Ohrarterie des Kaninchens auch nach der Excision grosser Stiicke aus dem Sympathicus und Auricularis magnas noch die von Schiff beschriebenen Sehwankungen des Lumens zeigt, so muss ich nach meinen zahlreichen Beobachtungen das Gegentheil behaupten; die nach der genannten Operation auftretenden geringfügigen Aenderungen im Lumen der arteriellen Gefässe entstehen vielmehr unter dem Einflusse mehr oder weniger rhythmischer Schwankungen des Blutdruckes, wie sie beim Kaninchen sehr häufig auftreten" ${ }^{3}$ ).

Fiir die Schwimmbat des Frosehes hat wohl Gunning ${ }^{4}$ ) zuerst Wiederkehr der Rhythmik nach Durchschneidung des Ischiadicus beschrieben, haben dann Riegel ${ }^{5}$ ) und Huizinga ${ }^{6}$ ) diese Angabe Saviotti ${ }^{7}$ ) gegenüber bestätigt. Allein schon Riegel glaubt

1) Schiff, Untersuchungen zur Physiol. des Nervensystems. Band I. p. 181. 1855 .

2) Vgl. auch Aubert, Hermann's Handb. der Physiol. Band IV. 1, p. 454. 1881 .

3) Sigm. Mayer, Hermann's Handb. d. Physiol. Bd. Y. 2, p. 478.

4) Gunning, Onderzoekingen over bloedsbeweging en stasis, Utrecht 1857 .

5) Riegel, Dies Archiv. 1871. Bd. IV. p. 353.

6) Huizinga, Dies Archiv. 1875. Bd. XI. p. 207.

7) Saviotti, Virchow's Archiv. 1870. Bd. L. p. 592. 
damit die Frage keineswegs zu Gunsten peripherer Ursache entschieden, könnten ja in solchen, keineswegs konstanten Fällen noch andere Bahnen den Verkehr zwischen Schwimmhaut und Centralmark vermitteln! Huizinga zwar meint allerdings mit einer Durchschneidung des Ischiadicus jeden Einfluss des Centralmarkes aufgehoben zu haben. Denn er findet reflectorischen Gefässkrampf wohl noch in der normalen, nicht aber jener Schwimmhaut, deren Ischiadicus durchschnitten war. Aber auch dieser Schluss ist näher betrachtet keineswegs bindend. Die wenigen anders verlaufenden Fasern dürften ja wohl genügen, die Rhythmik zu unterhalten, brauchten aber durchaus nicht auch noch im Stande zu sein, einen allgemeinen, überall gleichzeitig ausbrechenden Gefässkrampf zu erregen. Vielmehr würden solehe eben nur an ihren, von einander wohl sehr entfernten physiologischen Eintrittsstellen, also nur an wenigen, weit auseinandergelegenen Punkten eine erste Erregung setzen, und würde sich solche dann wohl im Sinne Engelmann's peristaltisch von Zelle zu Zelle weiter ausbreiten, es würde in solchem Falle aus einer reflectorischen Reizung also nur eine Verschiebung des Rhythmus, höchstens eine geringe Beschleunigung desselben resultiren können, scheint aber $\mathrm{Hu}$ izinga hierauf nicht besondere Aufmerksamkeit gerichtet zu haben.

Nach völliger Zerstörung des Centralmarkes hatte Nussba um ${ }^{1}$ ) Aufhören der Rhythmik beobachtet, hatten Gergens und Werber ${ }^{2}$ ) zwar noch Contractionen gesehen, wollen selber aber solche "wegen Mangels an Regelmässigkeit in Bezug auf die Zeit zwisehen dem jedesmaligen Eintreten von Erweiterung und Verengerung" nicht mit unserer Erscheinung identificiren. Gruppenbildungen des erstickenden Herzens ${ }^{3}$ ) könnten Aenderungen des Blutdruckes bedingen; Erstickung der Gefässwand könnte, wie bei jedem glattmuskeligen Organe, auch hier Erregungen einleiten; in dem speciell mitgetheilten Versuche war der Hüftnerv viele Tage vorher durchschnitten, im Sinne Schiff's könnten fibrilläre Zukkungen auch hier die Degeneration begleiten.

Endlich findet Huizinga in der verschieden frequenten Rhythmik benachbarter Arterien einen Grund für den localen

1) Nussbaum, Dies Archiv. 1875. Bd. X. p. 377.

2) Gergens \& Werber, Dies Archiv. 1876. Bd. XIII. p. 52.

3) Socoloff \& Luchsinger, Dies Archiv 1880, Bd. XXIII, p. 292. 
Charakter der Rhythmik. Allein, wer bürgt denn dafür, dass gerade in den centralen Stücken des Gefässapparates, die wir uns doch jetzt über das ganze Rückenmark vertheilt denken, überall eine einheitliche Entladung erfolgt, überall die gleiche Höhe der Erregbarkeit herrseht!

Fassen wir uns kurz! Für das Ohrherz der Kaninchen sprechen die Untersuchungen fast einstimmig zu Gunsten ausschliesslich centraler Innervation; für die Flügelherzen der Fledermänse sagt dasselbe dio einzige, überhaupt vorliegende Versuchsreihe Schiff's. Für die Arterien in der Schwimmhaut des Frosches sind die Stimmen zwar getheilt, aber auch hier können den Vertretern der localen Ursache der Rhythmik eine Reihe kräftiger Einwände entgegengehalten werden.

Auf der andern Seite aber darf man wohl daran festhalten, dass auch das Herz, das doch in sich selber den Sitz rhythmischer Entladungen trägt, morphologisch betrachtet eben Nichts Anderes ist als ein Gefässschlauch, der die stärkere Ausbildung seiner Wand ja nur gesteigerten mechanischen Anforderungen verdankt. Dann aber wird wie im Herzen so anch schon in der Gefässwand die Möglichkeit rhythmischer Erregung enthalten sein müssen.

Eine Reihe von Untersuchungen der letzten Jahre zeigten, wie bestimmte Bedingungen ehemischer, mechanischer, electrischer Reizung selbst in der ruhenden Herzwand Rhythmik erzeugten; Untersuchungen am Ureter einiger Säugethiere ${ }^{1)}$ zeigten auch hier in der mechanischen Spannung eine mächtige Ursache rhythmischer, rein peripherer Erregung. Sollte nicht auch ein Gefässschlauch der Herzspitze des Frosches, dem Harnschlauch des Hundes gleich sich rhythmisch verengen können, wenn dehnender Inhalt ihn reizt? Ich fasste wenigstens Hoffnung, unter bestimmten Bedingungen mindestens selbst unmittelbar nach der Trennung vom Centralmark der herrschenden Lehre entgegen Pulsationen der Gefässwand zu erzwingen.

Der Fledermausflugel bot ein schönstes, zudem noch so selten untersuchtes Object.

1) Vergl. einen folgenden Aufsatz. 


\section{Methode.}

Um ruhige Beobachtung zu sichern diente anfangs Aethernarkose, empfahl sich aber später als ganz ausgezeichnetes Mittel eine Gabe von 0,002 salzsaurem Morphium.

Zur Ausbreitung der Flughaut passte sehr gut der Emmert'sche, ursprünglich zur Beobachtung der Schwimmhaut des Frosches construirte Halter. Eine passend gelagerte Vene wird mit schwacher Vergrösserung beobachtet. (Hartnack Obj. IV., Ocul. II.)

\section{Versuehe.}

Versuehsform I. Gleich der erste Versuch bot ein überraschend günstiges Ergebniss. Der plex. brachialis war präparirt und durchschnitten. Die Venen der Flughaut aber pulsirten gleichwohl in kräftigster Weise; 8-10 Mal pro Minute. Sollte noch irgend ein feiner, mir nicht bekannter Nervenfaden verschont geblieben sein? Es wurden nun die nn. ulnaris, medianus, radialis am Handgelenk aufgesucht und durchschnitten, der Puls der Venen aber blieb immer noch bestehen; nun wurden die nebeneinander gelagerten zu- und abführenden Gefässe der Hand mit einem Häckchen emporgehoben und aller übrige Zusammenhang zwischen Hand und Körper mit kräftiger Scheere durchschnitten; die Rhythmik der Flügelvenen ist noch da! Nur feine sympathische Nervenplexus könnten jetzt allenfalls noch auf der Wand der Gefässe den Verkehr mit den Centren vermitteln. Eine tüchtige Bepinselung der Gefässe mit Ammoniak musste auch diese so oft eingewendeten Nebenwege vernichten; gleichwohl bleibt auch jetzt noch die Pulsation deutlich bestehen, zeigt höchstens unmittelbar nach dem Eingriffe eine geringe Schwäehung.

Die Pulsationen der Venen haben also wohl sicher periphere, locale Ursachen.

Der mitgetheilte Versuch sollte nur ein Beispiel sein. Eine Reihe anderer ergeben genau gleichen Befund; in einigen wenigen dagegen blieb für die ersten Minuten nach der Nervendurchschneidung der Puls allerdings aus, kam aber in kurzem von selbst immer wieder zurück. Aus Versuchsform IV aber werden wir deutlich ersehen, welche Momente hier mitspielen und im Anfange wenigstens den Puls allerdings unterdrücken könnten. 
Versuchsform II. Der Flügel ist wieder ausgespannt, eine gut pulsirende Vene zur Beobachtung eingestellt, der Arm gut fixirt. Nun wird mit möglichster Vermeidung von Verschiebungen der Flügel vom Thier abgeschnitten; rasch die vielleicht etwas verschobene Vene wieder ins Gesichtsfeld gebracht. Aber auch jetzt noch ist kräftige Pulsation der Venen in den ersten Minuten nach der Amputation sehr wohl zu sehen. Und hat endlich nach c. 4-5 Minuten die Rhythmik aufgehört, so ist die Vene auch vollkommen zusammengefallen, und man hat wohl das Recht, in dem völligen Verlust an Spannung den Grund des frühen Erlöschens der Pulsation zu vermuthen.

Versuchsform III. In anderen Versuchen wurde also der Fligel vorher stark ligirt, dann oberhalb des Bandes amputirt, die Venen der Flughaut also vor Verblutung geschititz. Jetzt pulsirten sie aber auch wesentlich länger, bis zu 10-15 Minuten.

Versuchsform IV. In eben erwähnter Versuchsform musste die Pulsation endlich aufhören, da die Gefässwand wohl allmählich erstickte. Es war nicht unwahrscheinlich, viel länger anhaltende Pulsationen im amputirten Flügel zu erbalten, wenn es möglich wäre, durch einen künstlichen Blutstrom die Lebenseigenschaften der Gefässwand zu erhalten.

Die Thiere wurden erstickt, eine Kanüle in die Aorta eingebunden, und c. 10 Minuten nach dem Tode geschlagenes, gut filtrirtes Ochsenblut aus einer Höhe von $40-50 \mathrm{~cm}$ in die Aorta geleitet. Bemerkenswerther Weise braucht das Blut durchaus nicht etwa vorher auf Körperwärme gebracht zu werden, besass dasselbe in allen Versuchen vielmehr nur Zimmertemperatur. Leicht stellte sich die Circulation wieder her, die Venen schwollen an, und traten nun Pulsationen auf, oft genug häufiger tnd kräftiger denn je. Ja selbst 20 Stunden nachdem Todedes Thieres zeigten die durch künstlichen Blutstrom durchströmten Venen ihrerhythmisebe Contraction indeutlichster $W$ eise. Sollte es nach den vorhergehenden Versuchsreihen iiberhaupt noch eines Beweises für die locale Ursache der Erscheinung bedürfen, in der That, derselbe wäre reichlich erbracht, wenn sich in den Venen der Flügel immer noch reges Leben erbalten lässt, zu einer Zeit, wo jegliche centrale Leistung schon längst erloschen, ja erhebliche Fäulniss des Thierrestes schon Platz gegriffen. - Anstatt Blut können wir sogar nur 
Salzwasser zur Durchströmung benutzen. Längere Zeit, über 50 Minuten zeigen sich auch so kräftige Contractionen der Wand. Sind diese endlich erloschen, so kann immer noch eine darauffolgende Durchströmung mit Blut mit Leichtigkeit „die Uhr des Lebens noch einmal aufziehen."

Die Versuche mit kïnstlicher Durchströmung aber lehrten noch mehr. Denn sie gestatteten in einfachster Weise, durch einfache Verstellung des Druckgefässes die Geschwindigkeit des Blutstroms, die Spannung der Venenwand zu ändern.

War der Druck Null, so hörte nach einigen Minuten die Pulsation auf, obschon noch immer Blut oder Salzwasser - allerdings in spärlicherem Maasse - durch die Vene floss; wurde der Druck auf 40-50 cm Wasser erhölht, so begannen nach kurzem die Venen anzuschwellen, erfolgte dann das rhythmische Spiel ihrer Wand. Sank endlich der Druck wieder auf Null, so sank auch die Pulszahl bis Null, aber nicht plötzlich; denn sie blieb für die nächsten Minuten noch auf geringer Höhe bestehen, bis eben das stark gefüllte Gefässsystem sich von der Ueberfüllung völlig entleert hatte. War endlich gegen Ende des Versuchs, bei völliger Ermattung der Gefässwand selbst bei hohem Drucke kein Puls mehr vorhanden, so konnte derselbe doch durch mehrmaliges Quetsehen des zuführenden Kautschulsschlauches oft genug noch erregt werden.

Wie beim Herzen und Ureter zeigt sich also auch hier beim Gefässschlauch in der mechanischen Dehnung der contractilen Wand ein mächtiger Reiz für die Erregung der Rhythmik.

Der Misserfolg Schiff's, so wie das auch in einigen unserer Versuche anfängliche Sistiren der Pulsationen werden auf solehen Mangel an Spannung zurückzuführen sein. Da die Arterien unmittelbar nach einer Nervendurehschneidung sich oft stark und anhaltend verengen ${ }^{1}$ ), so wird eine starke Entleerung der Venen und damit deren Pulslosigkeit leicht erklärlich.

Endlich geben diese Versuche noch einen weiteren, nicht unwichtigen biologischen Wink. Wenn es keiner besonderen Wärme des Blutes bedurfte, um gleichwobl noch so viele Stunden einige Lebenserscheinungen eines Warmblüters in kräftigster Weise

1) Vgl. Kendall \& Luchsinger, Dies Arch. 1876. Bd. XIII. p. 202. 
zu unterhalten, so zeigt dies deutlich genug, wie eben auch beim Warmblüter nicht alle Gewebe uiber einen Leisten zu schlagen sind, wie es eben auch beim Warmblüter Gewebe gibt, die gemäss ibrem Standort den Character und die Zähigkeit der Kaltblitter sich angeeignet haben.

Alle unsere Versuchsreihen erweisen in schönster Uebereinstimmung die Peripherie, die Gefässwand selber als Sitz ihrer rhythmischen Contraction. An unserem Objecte wenigstens prallen alle sonst wohl üblichen Einwände ab.

Wenn man oft schon unmittelbar, stets wenige Minuten nach der Durchschneidung der Nerven kräftige und äusserst regelmässige Pulsationen sieht, so ist an eine Erklärung dorch fibrilläre Zuckungen im Sinne Schiff's nicht mehr zu denken. Die oft gebrachten Zweifel nicht vollständiger Abtrennung des Centralnervensystems miissen unsern Amputationsversuchen gegenüber verstummen. Auch passive, durch Aenderungen des Blutdruckes etwa bedingte Schwankungen der Gefässweite sind nicht im Spiele, unsere Versuche mit künstlicher Durchströmung bezeugen dies evident.

Allerdings scheint unser Object auch ganz besonders günstig fiir die Entwicklung der Rhythmik zu sein. In den langen, engen Gefässen der Flughaut entwickeln sich ausserordentlich grosse Widerstände der Circulation. Dadurch wird in den Venen die vis a tergo ungemein vermindert, es müssten in kurzer Zeit Stauung'en eintreten. Aber ganz im Sinne von Pflitg er's ${ }^{1}$ ) teleologischem Causalgesetz birgt auch hier das Bedürfniss die Quelle seiner Befriedigung in sich. Die anschwellende Stauung der Vene beginnt endlich als mechanischer Reiz auf die Gefässwand zu wirken, trägt aber nun die erfolgende Contraction erfolgreichst bei zur Hebung des erschwerten Kreislaufs.

Den Sitz der peripheren Erregung näher zu bezeichnen dürfte gegenwärtig nicht möglich sein. Wenn man auch hergebrachter Weise gern an gangliöse Gebilde denken möchte, so ist dem-

1) Pflïge r, Die teleologische Mechanik der lebendigen Natur, Bonn 1877, p. 37. 
entgegen eben nur an die rhythmischen Pulsationen der ,ganglienfreien" Herzspitze zu erinnern.

Die Bedingungen der Rhythmik, ihre Veränderungen durch die verschiedensten äusseren Einflüsse festzustellen, wird Zweck weiterer Versuche sein müssen. Solche sind denn auch zusammen mit Freund und Collegen Hartmann bereits in Angriff genommen. Da Mangel an Material die Untersuchung aber in die Länge zu ziehen droht, seien jetzt schon einige gesicherte Resultate der gemeinschaftlichen Arbeit kurz angefügt.

Vor Allem wird die Bedeutung des Centralnervensystems interessiren. Denn wenn auch nach unseren jetzigen Kenntnissen die Rhythmik völlig unabhängig rom Centralnervensystem bestehen kann, so ist damit keineswegs ein regulirender Einfluss des Centralnervensystems auf den Ablauf der Rhythmik ausgeschlossen. Unsere Kenntnisse von der Herzinnervation lassen auch hier an beschleunigende und hemmende Wirkungen denken.

In unseren Versuchen wurde der n. ulnaris mit verschiedensten Reizarten und Reizstärken geprüft, es hat sich bis jetzt stets nur eine deutliche Beschleunigung (von 12 anf 16 p. m.), nie eine Verlangsamung gezeigt. Erinnert man sich aber der langen Diskussionen über die beiderlei Gefässnerven im n. ischiadicus, so wird man gleichwohl den Gedanken an hemmende Nerven noch keineswegs aufgeben müssen. Diese könnten ja sehr gut ebenfalls vorbanden, in ihrer Wirkung von den mitgereizten beschleunigenden Fasern nur iberstimmt sein.

Reizung der Nerven bedingt aber auch Aenderungen im arteriellen Blutstrome, damit Aenderungen in der Fuillung der Venen. Die dadurch indirect eingefïhrten Veränderungen der Erregung:sgrösse dürften nicht geringe Schwierigkeiten bereiten.

In sehr verständlicher Weise wirkt eine Erwärmung der Flughaut. Geringe Temperatursteigerungen beschleunigen die Rhythmik, höhere Temperaturen macken diastolischen Stillstand, der beim Wiederabkühlen ernenter Pulsation wieder weichen kann.

Sehr bedentende Beschleunigungen der Rhythmik sehen wir bei der Application tetanisirender Ströme.

Endlich wurde noch mit dem Studium der Gifte begonnen, vor Allem verdienen die als Herz- und Gefässgifte bekannten Agentien alle Beachtung.

Von all diesen Versuchen sei jetzt nur noch kurz der Wir- 
kungen des Amylnitrits gedacht. Ueberraschenderweise machte dies nämlich anfangs starke Beschleunigung und Verstärkung der Pulse, Stillstand aber erst kurz vor dem Tode des Thieres.

Sobald eine systematische Untersuchung all dieser Agentien beendet sein wird, soll eine ausführliche Darstellung folgen.

\section{Nachschrift.}

Kurz nach Einsendung vorstehenden Aufsatzes brachte ein Besuch von Herrn Professor Schiff in Bern die erwünschte Gelegenheit, die eben mitgetheilten Versuche mit ihm zu besprechen.

Zu meiner grossen Freude erfuhr ich denn, dass er jetzt seinen früheren Standpunkt aufgegeben hätte, ja zu Resultaten gelangt sei, die den meinigen in vielen Punkten glichen. Aber Sehiff hatte die Publication dieser seiner neuen Ergebnisse einstweilen noch verschieben wollen, bis die Ursache des von ihm öfter gefundenen Fehlens der Pulsation auch noch festgestellt wäre. Jetzt aber glaubte er damit nicht länger zurtickhalten zu dürfen, um selber möglichst bald die volle Uebereinstimmung unserer wesentlichen Ergebnisse zu bezengen.

Hoch erfreut iiber diese jetzt leider so selten gewordene Art wissenschaftlicher Discussion sende ich nun auch die folgenden Zeilen Schiff's auf dessen Wunsch zur Aufnahme ins Archiv.

„Die Versuche, über den Einfluss der Nerven auf den Venenpuls im Patagium der Fledermäuse, deren ich in meinen „Untersuchungen zur Physiologie des Nervensystems" Erwähnung gethan, wurden vor etwa 30 Jahren an tief ätherisirten Thieren angestellt. Ich hatte nur 2 Fledermäuse bekommen können. Der Plexus wurde durchschnitten und nachdem ich mich vom Stillstand der Pulsationen überzeugt, wurden die Thierchen rasch getödtet, ehe sie aus dem Aetherrausche völlig erwacht waren. Ich fürchtete, dass die frische Wunde in der Nähe der Schulter bei der zu einer erneuten Untersuchung nöthigen Ausspannung des Armes den erwachten Thieren schmerzhaft werden könnte.

Erst in den letzten Jahren kam ich auf diesen Gegenstand zurïck. Die Fortschritte der Wissenschaft in Betreff der Innervation der glatten Muskeln hatten immer mehr dargethan, dass wie ich es sehon in den erwähnten Untersuchungen für einige Gebiete 
erkannt hatte, der erste Erfolg einer Nervenlähmung sehr bald wieder einer erneuten, anfangs rasch zunehmenden, wenn auch stets etwas geschwächten oder in ihrer Form veränderten Bewegung weicht.

In meinen späteren Versuchen an Fledermäusen, die ich längere Zeit zu beobachten gedachte, verlegte ich die äussere Wunde hinter das Schulterblatt, so dass sie von der Ausspannung des Armes weniger gezerrt wurde, die Operation wurde rasch vollzogen und das Thier dann vertikal im Fokus eines horizontalen schwachen Mikroskopes von etwa $20 \mathrm{~cm}$ Objektdistanz aufgehängt. Ich fand:

1) Stillstand in der ersten Zeit nach der Operation. Diese erste Periode wird durch eine starke Zusammenziehung der Venen eingeleitet. Nachdem diese sich sehr bald wieder ausgedehnt, kann die erste Periode des Stillstandes 7 bis 15 Minuten danern. Sie hält selten über 10 Minuten an. Diese Zeit ist für eine primäre Lähmung sehr kurz, besonders wenn wir sie mit der Daner der primären Lähńnung im Ohrgefäss der Kaninchen vergleichen, aber ich kenne jetzt andere Organe, bei denen die Dauer nicht länger, ja noch kürzer ist.

2) Dann kommt eine kurze Periode, in der sich Zusammenziehung an einzelnen Stellen zeigt. Die Bewegung ist aber noch unregelmässig; ohne bestimmte Ordnung in ihrer Fortpflanzung. So kann sich ein Ast zur Hälfte zusammenziehen, ein Stiuck wird übersprungen, während der nächst höhere Stamm gleichzeitig in Contraktion geräth. Auch die Intensität, weniger die Dauer der Zusammenziehung ist an verschiedenen Stellen verschieden.

3) Die Contraktionen werden bald regelmässig und endlich sieht man, dass die Unregelmässigkeiten, nachdem sie sich auf immer engere Gebiete beschränkt haben, ganz verschwinden.

4) Wenn man jetzt die peripherischen Theile der durchschnittenen Nerven, die man mit Seidenfäden verschiedener Farbe umschlungen hatte, tetanisirt, so erhält man eine auffallend kurz danernde tetanische Zusammenziehung der Venen.

5) Unterbindung der Arterien und Venen lässt die Bewegung noch sehr lange (über eine Stunde) fortbestehen, ihr Umfang nimmt aber bald so ab, dass man nur bei scharfer Beobachtung einen Wechsel des Lumens erkennen kann. Man muss hier ein Mikroskopobjektiv von geringer Brennweite $(1 / 4$ bis $1 / 5$ engl. 
Zoll) wählen, das noch die Blutkörperehen als Körnchen erkennen lässt.

6) Wenn man nach wiederhergestellter Bewegung galvanokaustisch ein grosses (dreieckiges) Stück des Patagiums so ausschneidet, dass die Füllung der Gefässe möglichst erhalten bleibt, so dauert im isolirten Stiick die Gefässbewegung längere Zeit fort. Auch hier sieht man die Bewegung sehr bald geschwächt werden, aber die Verschiebung des Blutes bemerkt man noch sehr lange. Dieser Versuch gelingt bei Vespertilio murinus sehr leicht, aber er misslang mir an einer Plecotus auritus, an der ich ihn vor einigen Jahren in Florenz anstellen wollte.

7) Alle diese Versuche sind im Frühling und im Sommer angestellt. Ich habe niemals Fledermäuse im Winterschlaf überraschen können, und alle meine Versuche, Herbstthiere bis in den Winter aufzuheben misslangen. Aber vor 2 Jahren, während eines sehr milden Winterwetters fiel in Genf beim Reinigen eines grossen Amphitheaters eine grosse Rhinolophus zu Boden. Sie war vollständig wach und suchte zu entfliehen. Ich liess sie zu mir bringen, um den Schülern die Bewegung der Venen zu zeigen, aber trotz wohl erhaltener Cirkulation im Patagium war keine Spur von periodischer Gefüsscontraction zu sehen. Die Untersuchung: am folgenden Tage gab dasselbe Resultat. Ich wollte meine Veröffentlichung verschieben bis ich den Grund dieses sonderbaren Verhaltens gefunden. Wäre es vielleicht der Winterschlaf?" 